\title{
Starlike functions of complex order with bounded radius rotation by using quantum calculus
}

\author{
Asena Çetinkaya, Oya Mert
}

\begin{abstract}
In the present paper, we study on the subclass of starlike functions of complex order with bounded radius rotation using $q-$ difference operator denoted by $\mathcal{R}_{k}(q, b)$ where $k \geq 2, q \in(0,1)$ and $b \in \mathbb{C} \backslash\{0\}$. We investigate coefficient inequality, distortion theorem and radius of starlikeness for the class $\mathcal{R}_{k}(q, b)$.
\end{abstract}

\section{INTRODUCTION}

Let $\mathcal{A}$ be the class of functions $f$ of the form

$$
f(z)=z+\sum_{n=2}^{\infty} a_{n} z^{n},
$$

which are analytic in the open unit disc $\mathbb{D}:=\{z:|z|<1\}$ and satisfy the condition $f(0)=f^{\prime}(0)-1=0$ for every $z \in \mathbb{D}$. We say that $f_{1}$ is subordinate to $f_{2}$, written as $f_{1} \prec f_{2}$, if there exists a Schwarz function $\phi$ which is analytic in $\mathbb{D}$ with $\phi(0)=0$ and $|\phi(z)|<1$ such that $f_{1}(z)=f_{2}(\phi(z))$. In particular, when $f_{2}$ is univalent, then the subordination is equivalent to $f_{1}(0)=f_{2}(0)$ and $f_{1}(\mathbb{D}) \subset f_{2}(\mathbb{D})$ (Subordination principle $[4]$ ).

In 1909 and 1910, Jackson [5, 6] initiated a study of $q$ - difference operator by

$$
D_{q} f(z)=\frac{f(z)-f(q z)}{(1-q) z}, \quad \text { for } z \in B \backslash\{0\},
$$

where $B$ is a subset of complex plane $\mathbb{C}$, called $q-$ geometric set if $q z \in B$, whenever $z \in B$. Note that if a subset $B$ of $\mathbb{C}$ is $q-$ geometric, then it contains all geometric sequences $\left\{z q^{n}\right\}_{0}^{\infty}, z q \in B$. Obviously, $D_{q} f(z) \rightarrow$ $f^{\prime}(z)$ as $q \rightarrow 1^{-}$. Note that such an operator plays an important role in the theory of hypergeometric series and quantum physics (see for instance $[1,3,7])$.

2010 Mathematics Subject Classification. 30C45.

Key words and phrases. $q$-starlike function of complex order, bounded radius rotation, coefficient inequality, distortion theorem, radius of starlikeness.

Full paper. Received 14 September 2018, revised 4 December 2018, accepted 8 December 2018, available online 15 December 2018. 
For a function $f(z)=z^{n}$, we observe that

$$
D_{q} z^{n}=\frac{1-q^{n}}{1-q} z^{n-1} .
$$

Therefore we have

$$
D_{q} f(z)=1+\sum_{n=2}^{\infty} a_{n} \frac{1-q^{n}}{1-q} z^{n-1},
$$

where $[n]_{q}=\frac{1-q^{n}}{1-q}$. Clearly, as $q \rightarrow 1^{-},[n]_{q} \rightarrow n$.

Denote by $\mathcal{P}_{q}$ the family of functions of the form $p(z)=1+p_{1} z+p_{2} z^{2}+$ $p_{3} z^{3}+\ldots$, analytic in $\mathbb{D}$ and satisfying the condition

$$
\left|p(z)-\frac{1}{1-q}\right| \leq \frac{1}{1-q},
$$

where $q \in(0,1)$ is a fixed real number.

Lemma $1.1([2]) . p \in \mathcal{P}_{q}$ if and only if $p(z) \prec \frac{1+z}{1-q z}$. This result is sharp for the functions $p(z)=\frac{1+\phi(z)}{1-q \phi(z)}$, where $\phi$ is a Schwarz function.

A function $p$ analytic in $\mathbb{D}$ with $p(0)=1$ is said to be in the class $\mathcal{P}_{k}(q)$, $k \geq 2, q \in(0,1)$ if and only if there exists $p_{1}^{(1)}, p_{2}^{(2)} \in \mathcal{P}_{q}$ such that

$$
p(z)=\left(\frac{k}{4}+\frac{1}{2}\right) p_{1}^{(1)}(z)-\left(\frac{k}{4}-\frac{1}{2}\right) p_{2}^{(2)}(z) .
$$

For $q \rightarrow 1^{-}, \mathcal{P}_{k}(q) \equiv \mathcal{P}_{k}$, (see [10]); for $k=2, q \rightarrow 1^{-}, \mathcal{P}_{k}(q) \equiv \mathcal{P}$ is the well known class of functions with positive real part. Also, for $k=2, \mathcal{P}_{k}(q) \equiv \mathcal{P}_{q}$ consists of all functions subordinate to $\frac{1+z}{1-q z}, z \in \mathbb{D}$.

Definition 1.1. Let $f$ of the form (1) be an element of $\mathcal{A}$. If $f$ satisfies the condition

$$
z \frac{D_{q} f(z)}{f(z)}=p(z), \quad p \in \mathcal{P}_{k}(q),
$$

with $k \geq 2, q \in(0,1)$, then $f$ is called $q-$ starlike function with bounded radius rotation denoted by $\mathcal{R}_{k}(q)$. This class was introduced and studied by Noor et al. [9].

Definition 1.2. Let $f$ of the form (1) be an element of $\mathcal{A}$. If $f$ satisfies the condition

$$
1+\frac{1}{b}\left(z \frac{D_{q} f(z)}{f(z)}-1\right)=p(z), \quad p \in \mathcal{P}_{k}(q),
$$

with $k \geq 2, q \in(0,1), b \in \mathbb{C} \backslash\{0\}$, then $f$ is called $q$ - starlike function of complex order with bounded radius rotation denoted by $\mathcal{R}_{k}(q, b)$. When $q \rightarrow 1^{-}, b=1$, the class $\mathcal{R}_{k}(q, b)$ reduces to $\mathcal{R}_{k}$ (see [10]). For $k=2, q \rightarrow 1^{-}$, the class $\mathcal{R}_{k}(q, b)$ reduces to $\mathcal{S}^{*}(1-b)$ (see [8]). For $k=2, q \rightarrow 1^{-}, b=1$ the class $\mathcal{R}_{k}(q, b)$ reduces to traditional class of the starlike functions $\mathcal{S}^{*}$. 
We investigate coefficient inequality, distortion theorem and radius of starlikeness for the class $\mathcal{R}_{k}(q, b)$.

\section{Main Results}

We first prove coefficient inequality for the class $\mathcal{R}_{k}(q, b)$. For our main theorem, we need the following lemma.

Lemma 2.1 ([11]). Let $p(z)=1+p_{1} z+p_{2} z^{2}+\ldots$ be an element of $\mathcal{P}_{k}(q)$, then

$$
\left|p_{n}\right| \leq \frac{k}{2}(1+q)
$$

This result is sharp for the functions

$$
p(z)=\left(\frac{k}{4}+\frac{1}{2}\right) p_{1}^{(1)}(z)-\left(\frac{k}{4}-\frac{1}{2}\right) p_{2}(z)^{(2)},
$$

where $p_{1}^{(1)}, p_{2}^{(2)} \in \mathcal{P}_{q}$.

Theorem 2.1. If $f \in \mathcal{R}_{k}(q, b)$, then

$$
\left|a_{n}\right| \leq \frac{1}{\left([n]_{q}-1\right) !} \prod_{\nu=1}^{n-1}\left(\left([\nu]_{q}-1\right)+\frac{k}{2}|b|(1+q)\right) .
$$

This inequality is sharp for every $n \geq 2$.

Proof. In view of definition of the class $\mathcal{R}_{k}(q, b)$ and subordination principle, we can write

$$
1+\frac{1}{b}\left(z \frac{D_{q} f(z)}{f(z)}-1\right)=p(z)
$$

where $p \in \mathcal{P}_{k}(q)$ with $p(0)=1$. Since $f(z)=z+\sum_{n=2}^{\infty} a_{n} z^{n}$ and $p(z)=$ $1+p_{1} z+p_{2} z^{2}+\ldots$, then we have

$$
\begin{aligned}
& z+[2]_{q} a_{2} z^{2}+[3]_{q} a_{3} z^{3}+[4]_{q} a_{4} z^{4}+\ldots \\
= & z+\left(a_{2}+b p_{1}\right) z^{2}+\left(a_{3}+b p_{1} a_{2}+b p_{2}\right) z^{3}+ \\
& \left(a_{4}+b p_{1} a_{3}+b p_{2} a_{2}+b p_{3}\right) z^{4}+\ldots
\end{aligned}
$$

Comparing the coefficients of $z^{n}$ on both sides, we obtain

$$
[n]_{q} a_{n}=a_{n}+b p_{1} a_{n-1}+b p_{2} a_{n-2}+\cdots+b p_{n-2} a_{2}+b p_{n-1}
$$

for all integer $n \geq 2$. In view of Lemma 2.1, we get

$$
\left([n]_{q}-1\right)\left|a_{n}\right| \leq \frac{k}{2}|b|(1+q)\left(\left|a_{n-1}\right|+\cdots+\left|a_{2}\right|+1\right),
$$

or equivalently

$$
\left|a_{n}\right| \leq \frac{\frac{k}{2}|b|(1+q)}{[n]_{q}-1} \sum_{\nu=1}^{n-1}\left|a_{\nu}\right|, \quad\left|a_{1}\right|=1 .
$$


In order to prove (2), we will use process of iteration. Let $c=\frac{k}{2}|b|(1+q)$ and use our assumption $\left|a_{1}\right|=1$ in (3), we obtain successively

$$
\begin{aligned}
& \text { for } n=2, \quad\left|a_{2}\right| \leq \frac{1}{[2]_{q}-1} c, \\
& \text { for } n=3, \quad\left|a_{3}\right| \leq \frac{1}{\left([3]_{q}-1\right) !} c\left(\left([2]_{q}-1\right)+c\right), \\
& \text { for } n=4, \quad\left|a_{4}\right| \leq \frac{1}{\left([4]_{q}-1\right) !} c\left(\left([2]_{q}-1\right)+c\right)\left(\left([3]_{q}-1\right)+c\right) .
\end{aligned}
$$

Hence induction shows that for $n$, we obtain

$$
\left|a_{n}\right| \leq \frac{1}{\left([n]_{q}-1\right) !} c\left(\left([2]_{q}-1\right)+c\right)\left(\left([3]_{q}-1\right)+c\right) \cdots\left(\left([n-1]_{q}-1\right)+c\right) .
$$

This proves (2).

This inequality is sharp, because extremal function is the solution of the $q-$ differential equation

$$
1+\frac{1}{b}\left(z \frac{D_{q} f(z)}{f(z)}-1\right)=\left(\frac{k}{4}+\frac{1}{2}\right) \frac{1+z}{1-q z}-\left(\frac{k}{4}-\frac{1}{2}\right) \frac{1-z}{1+q z} .
$$

Corollary 2.1. Taking $q \rightarrow 1^{-}$and choosing $k=2, b=1$ in (2), we get $\left|a_{n}\right| \leq n$ for every $n \geq 2$. This is well known coefficient inequality for starlike functions.

We now introduce distortion theorem and radius of $q-$ starlikeness for the class $\mathcal{R}_{k}(q, b)$.

Lemma $2.2([9])$. Let $f \in \mathcal{R}_{k}(q)$. Then for $k \geq 2$ and $q \in(0,1)$, we have

$$
\left|z \frac{D_{q} f(z)}{f(z)}-\frac{1+q r^{2}}{1-q^{2} r^{2}}\right| \leq \frac{\frac{k}{2}(1+q) r}{1-q^{2} r^{2}} .
$$

Theorem 2.2. If $f$ is an element of $\mathcal{R}_{k}(q, b)$, then

(4) $(r F(k, q, \operatorname{Re} b,|b|,-r))^{\frac{1-q}{\log q^{-1}}} \leq|f(z)| \leq(r F(k, q, \operatorname{Re} b,|b|, r))^{\frac{1-q}{\log q^{-1}}}$,

where

$$
F(k, q, \operatorname{Re} b,|b|, r)=\frac{(1+q r)^{\frac{1+q}{q}\left(\frac{k}{4}|b|-\frac{1}{2} \operatorname{Re} b\right)}}{(1-q r)^{\frac{1+q}{q}\left(\left(\frac{k}{4}|b|+\frac{1}{2} \operatorname{Re} b\right)\right.} .}
$$

This bound is sharp.

Proof. In view of Lemma 2.2 and subordination principle, we write

$$
\left|1+\frac{1}{b}\left(z \frac{D_{q} f(z)}{f(z)}-1\right)-\frac{1+q r^{2}}{1-q^{2} r^{2}}\right| \leq \frac{\frac{k}{2}(1+q) r}{1-q^{2} r^{2}} .
$$


Therefore, after routine calculations, we get

$$
\left|z \frac{D_{q} f(z)}{f(z)}-\frac{1+\left(b\left(q+q^{2}\right)-q^{2}\right) r^{2}}{1-q^{2} r^{2}}\right| \leq \frac{\frac{k}{2}|b|(1+q) r}{1-q^{2} r^{2}} .
$$

After calculations in (5), we obtain

$$
\begin{aligned}
& \operatorname{Re}\left(z \frac{D_{q} f(z)}{f(z)}\right) \leq \frac{1+\frac{k}{2}|b|(1+q) r+\left(\left(q^{2}+q\right) \operatorname{Re} b-q^{2}\right) r^{2}}{(1-q r)(1+q r)}, \\
& \operatorname{Re}\left(z \frac{D_{q} f(z)}{f(z)}\right) \geq \frac{1-\frac{k}{2}|b|(1+q) r+\left(\left(q^{2}+q\right) \operatorname{Re} b-q^{2}\right) r^{2}}{(1-q r)(1+q r)} .
\end{aligned}
$$

On the other hand, we have

$$
\operatorname{Re}\left(z \frac{D_{q} f(z)}{f(z)}\right)=r \frac{\partial_{q}}{\partial r} \log |f(z)| \text {. }
$$

Considering (6) and (7) together, respectively, we get

$$
\begin{aligned}
& \frac{\partial_{q}}{\partial r} \log |f(z)| \leq \frac{1}{r}+\frac{(1+q)\left(\frac{1}{2} \operatorname{Re} b+\frac{k}{4}|b|\right)}{(1-q r)}-\frac{(1+q)\left(\frac{1}{2} \operatorname{Re} b-\frac{k}{4}|b|\right)}{(1+q r)}, \\
& \frac{\partial_{q}}{\partial r} \log |f(z)| \geq \frac{1}{r}+\frac{(1+q)\left(\frac{1}{2} \operatorname{Re} b-\frac{k}{4}|b|\right)}{(1-q r)}-\frac{(1+q)\left(\frac{1}{2} \operatorname{Re} b+\frac{k}{4}|b|\right)}{(1+q r)} .
\end{aligned}
$$

Taking $q-$ integral on both sides of the last inequalities, we get (4).

This bound is sharp, because extremal function is the solution of the $q-$ differential equation

$$
1+\frac{1}{b}\left(z \frac{D_{q} f(z)}{f(z)}-1\right)=\left(\frac{k}{4}+\frac{1}{2}\right) \frac{1+z}{1-q z}-\left(\frac{k}{4}-\frac{1}{2}\right) \frac{1-z}{1+q z} .
$$

Corollary 2.2. Taking $q \rightarrow 1^{-}$and $b=1$ in Theorem 2.2, we get the following well known result:

$$
\frac{z(1-z)^{\left(\frac{k}{2}-1\right)}}{(1+z)^{\left(\frac{k}{2}+1\right)}} \leq|f(z)| \leq \frac{z(1+z)^{\left(\frac{k}{2}-1\right)}}{(1-z)^{\left(\frac{k}{2}+1\right)}} .
$$

Let $f \in \mathcal{A}$, then the real number

$$
r^{*}(f)=\sup \left\{r>0, \operatorname{Re}\left(z \frac{D_{q} f(z)}{f(z)}\right)>0 \quad \text { for all } z \in \mathbb{D}\right\}
$$

is called the starlikeness of the class $\mathcal{A}$. The second inequality of (6) gives the starlikeness of the class $\mathcal{R}_{k}(q, b)$ as below:

$$
r^{*}(f)=\frac{k|b|(1+q)-\sqrt{k^{2}|b|^{2}(1+q)^{2}-16\left(\left(q^{2}+q\right) \operatorname{Re} b-q^{2}\right)}}{4\left(\left(q^{2}+q\right) \operatorname{Re} b-q^{2}\right)} .
$$

If $q \rightarrow 1^{-}, b=1$, then this radius reduces to $r^{*}(f)=\frac{k-\sqrt{k^{2}-4}}{2}$. This is the radius of the class $\mathcal{R}_{k}$ which was obtained by Pinchuk (see [10]). For $q \rightarrow 1^{-}, k=2$, we get the starlikennes of starlike functions of complex order 
as $r_{f}^{*}=\frac{|b|-\sqrt{|b|^{2}-2 \operatorname{Re} b+1}}{2 \operatorname{Re} b-1}$. If $k=2, b=1$ then the radius of the starlikeness of the class $\mathcal{S}_{q}^{*}$ is $r^{*}(f)=\frac{1}{q}$.

\section{REFERENCES}

[1] G. E. Andrews, Applications of basic hypergeometric functions, SIAM Rev., 16 (4) (1974), 441-484.

[2] A. Çetinkaya, O. Mert, A certain class of harmonic mappings related to functions of bounded boundary rotation, Proc. of $12^{\text {th }}$ Int. Sym. on Geometric Function Theory and Applications, (2016), 67-76.

[3] N. J. Fine, Basic Hypergeometric series and applications, Math. Surveys Monogr., 1988.

[4] A. W. Goodman, Univalent functions volume I and II, Polygonal Pub. House, 1983.

[5] F. H. Jackson, On q- functions and a certain difference operator, Trans. Royal Soc. Edinburgh, 46 (2) (1909), 253-281.

[6] F. H. Jackson, On q- definite integrals, Quart. J. Pure Appl. Math., 41 (1910), 193-203.

[7] V. Kac, P. Cheung, Quantum calculus, Springer, 2002.

[8] M. A Nasr, M. K. Aouf, Starlike functions of complex order, J. Natur. Sci. Math., 25 (1) (1985), 1-12.

[9] K. I. Noor, M. A. Noor, Linear combinations of generaized q- starlike functions, Appl. Math. Inf. Sci., 11 (3) (2017), 745-748.

[10] B. Pinchuk, Functions of bounded boundary Rotation, Isr. J. Math., 10 (1) (1971), 6-16.

[11] Y. Polatoglu, A. Çetinkaya, O. Mert, Some properties of the analytic functions with bounded radius rotation, Creative Mathematics and Informatics, 28 (1) (2019), 6 pages.

\section{Asena Çetinkaya}

Department of Mathematics and Computer Sciences ISTANBUL KÜLTÜR UNIVERSITY

ISTANBUL

TURKey

E-mail address: asnfigen@hotmail.com

\section{OYA Mert}

Department of Basic Sciences

Altinbaş University

IsTANBUL

TURKEY

E-mail address: oya.mert@altinbas.edu.tr 